\title{
EINE INSCHRIFT VON TI. CLAUDIUS PAULINUS AUS PANNONIEN? ABSCHRIFT EINER RÖMERZEITLICHEN BASE ODER EINE NEUZEITLICHE FÄLSCHUNG
}

\author{
P. KOVÁCS* - P. PROHÁSZKA** \\ * Pázmány Péter Katholische Universität, H-2081 Piliscsaba, Egyetem u. 1. \\ E-Mail: kovacs.peter@btk.ppke.hu \\ ** H-2500 Esztergom, József Attila tér 2. \\ E-Mail: prohaszkapeter@freemail.hu
}

\begin{abstract}
An Inscription of Ti. Claudius Paulinus from Pannonia? A drawing of an unpublished Roman statue base from Tát came to light together with several archaeological (mainly medieval) finds in a $19^{\text {th }}$-century (?) booklet in Esztergom, according to the record the drawing was prepared in 1814. The inscription of the statue base describes a senatorial career and the person can be identified most probably with Ti. Claudius Paulinus, governor of Lower Britain in 220 (PIR2 II 231 Nr. 955 cf. RIB 311, 1280 , CIL XIII 3162). As the text follows almost exactly his earlier inscription from Britain (RIB 311) and it contaminates a Pannonian funerary text from Sárisáp (RIU 769), it is highly likely that the drawing is a $20^{\text {th }}$-century forgery.
\end{abstract}

Keywords: Research history of Latin epigraphy in Pannonia, forgery, Romain Britain

Bonae memoriae Univ. Prof. Árpád Szállási

Während eines Gesprächs im Frühjahr 2011 erwähnte der im Vorjahr verstorbene berühmte Medizinhistoriker Dr. Árpád Szállási, dass er ein Zeichnungsbuch besitze, das interessante und bemerkenswerte Zeichnungen beinhaltet. ${ }^{1}$ Er erwarb es im Antiquariat zu Esztergom in den 1960er Jahren. Dank seiner Gefälligkeit konnten wir dieses Buch untersuchen und die Zeichnungen einscannen. Das Format des Buches ist 19,5 $\times 21,5 \mathrm{~cm}$ und es besteht aus 40 Blättern. Auf 16 Seiten kommen Zeichnungen, Eintragungen und eingeklebte Papiere vor. Auf der ersten Seite befinden sich die Eintragungen: „Ex Bibliothec Horváth Mihály. A.D. 1847“, „Pulszky Pro mem. IX. 26. 1848“ sowie mit Bleistift „Csernoch János püspök úré volt 1908-ban“ (Es gehörte 1908 dem Herrn Bischof János Csernoch). Die zweite Seite trägt noch eine Eintragung: „Original Skizzen Pro memoriam (sic!) Horváth Mihály. In nomine Dei." Leider konnten wir über die Person von Mihály Horváth keine biographischen Angaben finden. Auf manchen Seiten sind Zeichnungen archäologischer Funde - wie zum Beispiel der Funde des 10. Jahrhunderts aus Pomáz oder die Münzen eines mittelalterlichen Schatzes aus Esztergom - bzw. über die 1848 freigelegten Königsgräber von Székesfehérvár. Neben den Zeichnungen befinden sich im Buch eingeklebte Papiere, wie zum Beispiel eine Stadtkarte von Pest bzw. ein Probeabdruck eines Notgeldes von Rozsnyó aus dem Jahr 1849. Zu diesen gehört auch ein Blatt von der Größe 17,50×24,70 cm, was zusammengefaltet eingeklebt war. Das Papier und die Buchstaben sind für das 19. Jahrhundert charakteristisch. Auf dem Blatt befindet sich eine Bleistift Zeichnung einer römerzeitlichen Inschrift zu sehen. Darunter steht die folgende Anmerkung mit Tinte geschrieben: „Ezen kő a’ régi időkből Táth-nál került elő a' régi tsárda bontásnál, és ma is ottan álla. Rajzolta híven: Radvánszky Pál.

${ }^{1}$ Wir sind Herrn Dr. Árpád Szállási für die Bearbeitung und Veröffentlichung der Zeichnungen zu Dank verpflichtet. An dieser stelle möchten wir Dr A. R. Birley und Herrn Prof. Dr. W. Eck für ihre DOI: 10.1556/AArch.65.2014.1.5
Hilfe und Ratschläge danken. Für zahlreiche Verbesserungen bei den deutschen Formulierungen des Beitrages sind wir Prof. Dr. Emmeig (Wien) zu Dank verpflichtet.

Acta Archaeologica Academiae Scientiarum Hungaricae 65 (2014) 131-137 0001-5210/\$20.00 C 2014 Akadémiai Kiadó, Budapest 
Esztergom, 1814. Októb 7.“ (Dieser Stein aus den alten Zeiten kam bei Tát während Abrissarbeiten des alten Dorfkrugs zum Vorschein und steht heute auch da. Getreulich zeichnete: Pál Radvánszky. Esztergom, 7. Oktob. 1814.) Leider konnten wir über die Person von Pál Radvánszky keine biographischen Angaben finden. Die Fundstelle des Steins ist sehr interessant, weil der Historiker Lajos Némethy, über einen römischen Stein neben der Táter Landstraße am Anfang des 20. Jahrhunderts auch berichtete. ${ }^{2}$ Die Inschrift dieses Steins wurde leider weder von Némethy, noch von anderen abgeschrieben oder veröffentlicht.

Nach der Zeichnung handelt es sich um eine Base, die oben abgebrochen, rechts beschädigt ist (Abb. 1). Das Inschriftfeld ist von einem doppelt profilierten Rahmen umgeben. Die Interpunktionen sind meistens mit Doppelpunkten markiert.

Aufgrund der Zeichnung kann die folgende Inschrift rekonstruiert werden:

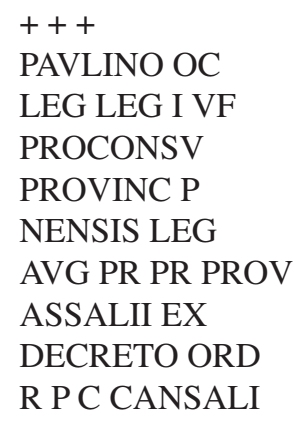

Auf den ersten Blick kann festgestellt werden, dass es sich um eine Kontamination zweier verschiedener Inschriften handelt und mehrere Buchstaben sicherlich falsch wiedergegeben sind. Die erste Inschrift ist eine Statuenbase mit senatorischer Laufbahn, die zweite gehört zu einer Grabinschrift aus Sárisáp, die nicht weit von Tát im Jahre 1901 in ein barockes Bad (gebaut im Jahre 1785) eingemauert gefunden wurde (RIU 769) (Abb. 2). ${ }^{3}$ Die Namen Assalii und Cansali stammen wohl aus dieser Stele: Aicca Cansali f(ilia) / Asalia an(n)oru/m XL Racio uxo/ ris su[]ae titulum / posit (!). Cansulus und seine Tochter Aicca gehörten zum illyrischen Stamm der Azalier, die in Nordpannonien in der Gegend von Brigetio lebten. ${ }^{4}$

Viel interessanter ist die erste Inschrift, die den Namen und die Laufbahn eines römischen Senators (bzw. den Namen einer Stadt, die die Statue errichtet hat) erwähnt. In der ersten Zeile sind drei beschädigte Buchstaben zu sehen: Der erste scheint ein D, der zweite ein O oder ein C zu sein, der dritte hat zwei vertikale Hasten. Das zweite $\mathrm{O}$ in der zweiten Zeile kann nur als ein $\mathrm{C}$ interpretiert werden, weil es nur zur Abkürzung $v$ (ir) $c$ (larissimus) gehören kann. Das letzte P der Zeile 4 muss in ein N emendiert werden, weil es nur zum Provinznamen Narbonensis gehören kann. Anhand dieser Korrektur kann die Inschrift aufgelöst werden, wie folgt:

$+++/$ Paulino v(iro) c(larissimo) / leg(ato) leg(ionis) I VF / proconsu[li]) / provinciae) N[arbo]/nensis leg(ato) / Aug(usti) pr(o) pr(aetore) prov(inciae) / ASSALII ex / decreto ord(inis) r(es) / p(ublica) CANSALI.

Der Name der zweiten Provinz und Stadt gehört zu der oben genannten Grabinschrift von Sárisáp (RIU 769). Paulinus kann aufgrund seines Cursus honorum höchstwahrscheinlich mit Ti. Claudius Paulinus identifiziert werden (PIR ${ }^{2}$ II 231 Nr. 955 cf. RIB 311, 1280, CIL XIII 3162), dessen Laufbahn unserer Inschrift entspricht bzw. die beschädigten und offensichtlich falsch gelesenen Buchstaben der Zeile 1 auch zu T CL korrigiert werden können: ${ }^{5}$

214-215 n. Chr. legatus legionis II Augustae

216 n. Chr. Statthalter der Provinz Gallia Narbonensis

218 n. Chr. Statthalter der Provinz Gallia Lugdunensis

220 n. Chr. Statthalter der Provinz Britannia inferior

Mit Hilfe seines Cursus kann auch der Name der Legion in Zeile 3 emendiert werden: legio II A[ug(usta)?]. Man könnte es auch als leg(io) I a[d(iutrix)] lesen: So war Paulinus auch der Kommandeur der Legion von Brige1979,324

2 NÉMETHY 1906, 188; vgl. Horváth-KELEMEN-TORMA 368, Nr. $19 / 17$.

${ }^{3}$ Zum Fundort siehe HoRváth-KelEMEN-ToRma 1979,

Acta Archaeologica Academiae Scientiarum Hungaricae 65, 2014
${ }^{4}$ Mócsy $1959,54-57$.

${ }^{5}$ Pflaum 1978, 43-44; Birley 1982, 537, n. 22; HalfMANN 1982, 635; LEUNISSEN 1989, 271, 288, 304, 338, 396; BIRLEY 2005, 342-344. 


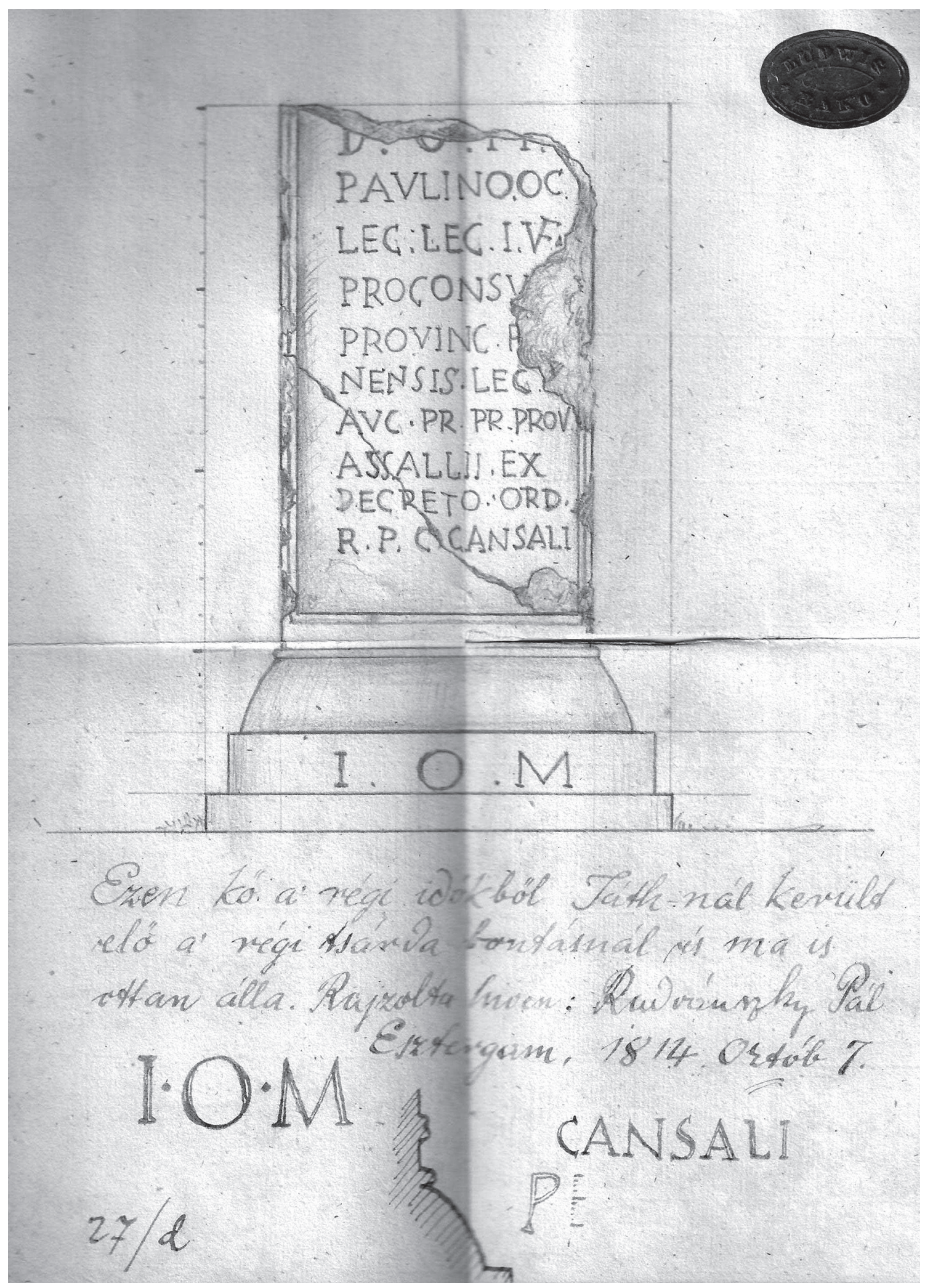

Abb. 1. Zeichnung der Base im Notizbuch von M. Horváth (Privatbesitz) 


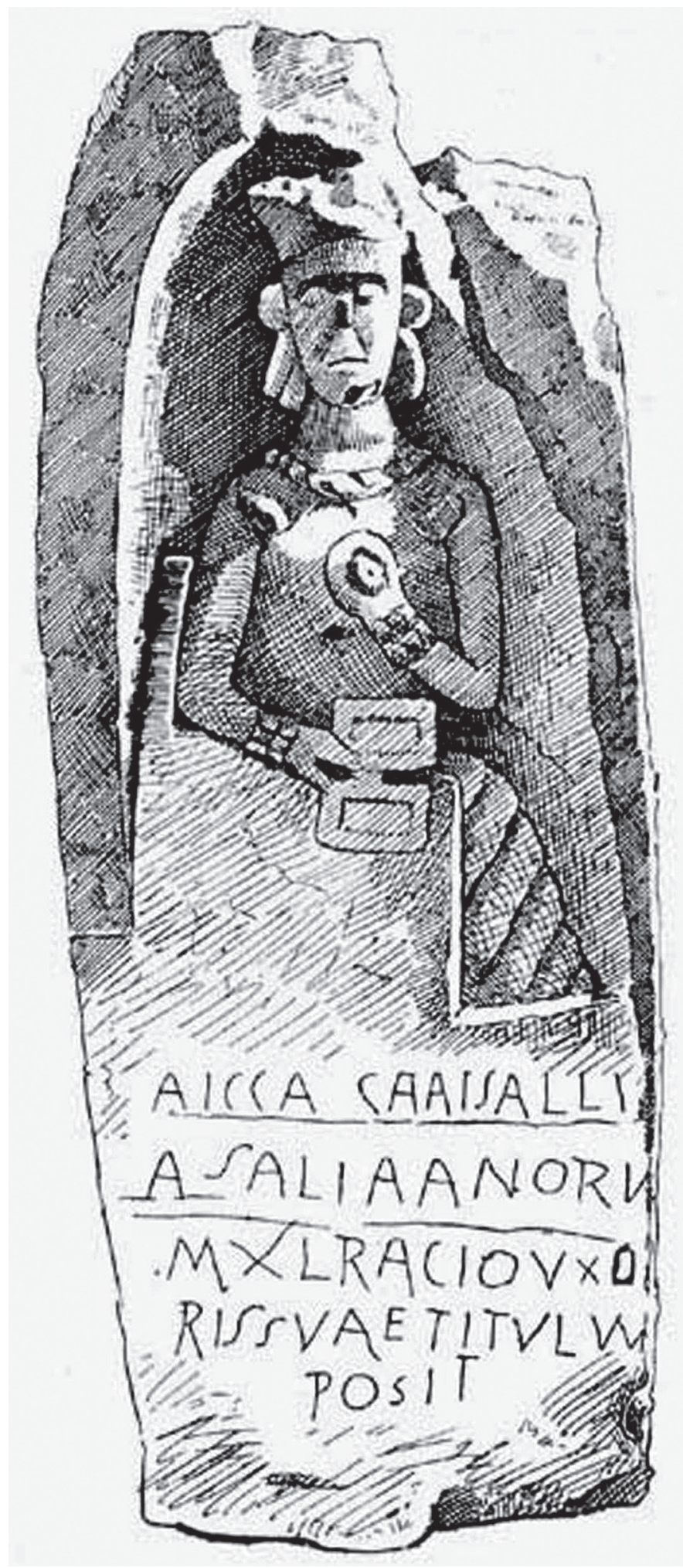

Abb. 2. Zeichnung des Grabsteins aus Sárisáp (RIU 769) 


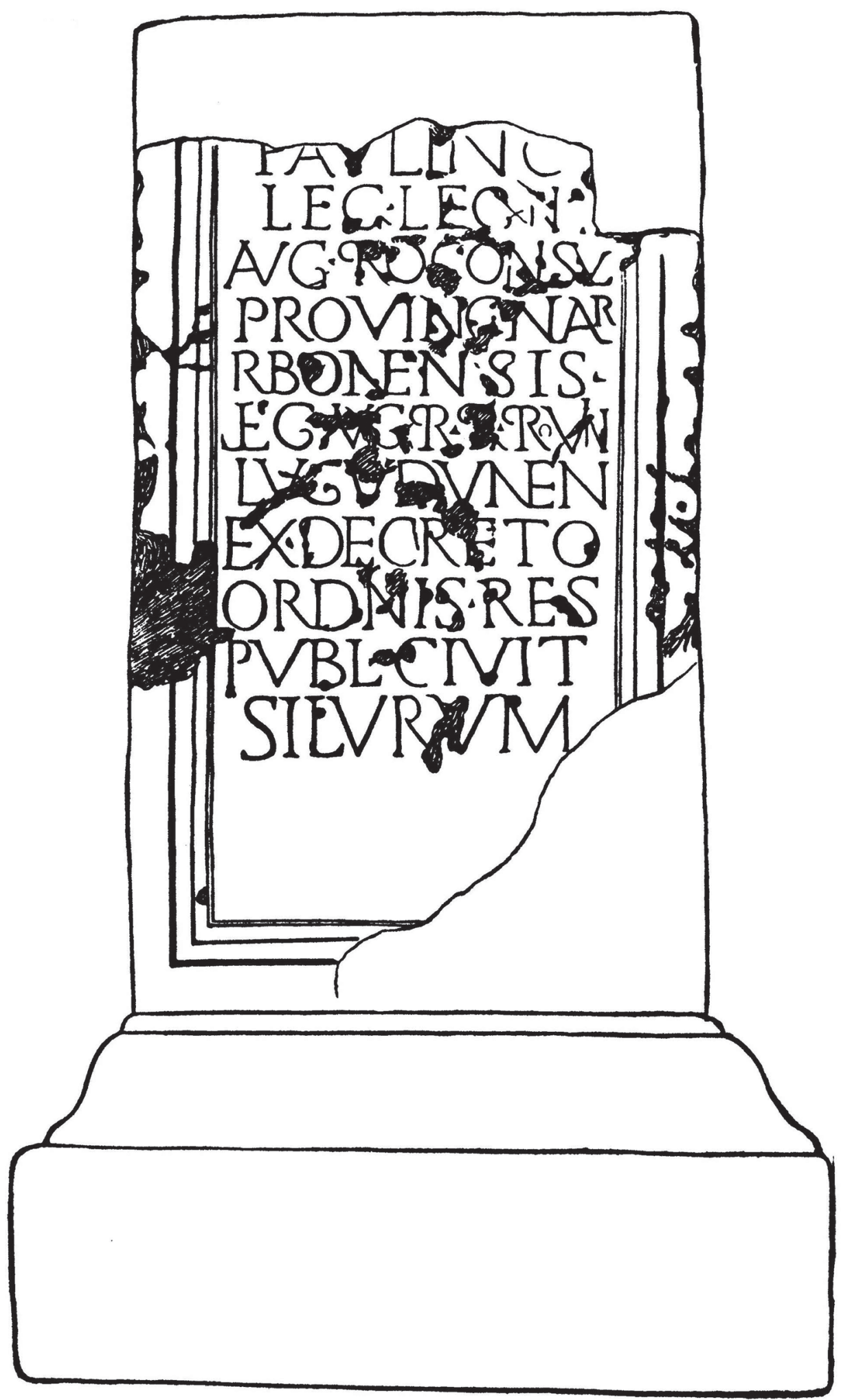

Abb. 3. Inschrift des Paulinus aus Caerwent (RIB 311) 
tio. Damit könnte man seine Statuenbase in Pannonien erklären. In diesem Fall kann unsere Inschrift folgendermaBen rekonstruiert werden:

Ti(berio) Cl(audio) / Paulino v(iro) c(larissimo) / leg(ato) leg(ionis) II A[ug(ustae) oder-d(iutricis)?] / proconsu[li]]) / provinciae) N[arbo]/nensis leg(ato) / Aug(usti) pr(o) pr(aetore) prov(inciae) / Lugdun(ensis) ex / decreto ord(inis) r(es) / p (ublica) CANSALI.

Unten kann man wohl die Corona eines Altars sehen, die sicherlich nicht zur Base gehörte. Die Dedikation, die erste Zeile der Inschrift, befindet sich hier auf dem Gesims:

I(ovi) O(ptimo) M(aximo)

- - - -

Die Buchstaben IOM sowie der Querschnitt der Base wurden unten nochmals und genauer nachgezeichnet.

Tát ist ein bekannter römerzeitlicher Fundort in Pannonien, der entlang der Limesstraße zwischen Brigetio und Aquincum lag. ${ }^{6}$ In Tát war früher nur eine Inschrift - ein dem Hercules gewidmeter Altar - bekannt, die im 19. Jahrhundert ante aedes Francisci Somogyi $n .97$ lag, seither aber leider verschollen ist (CIL III $13391=$ RIU 760). Diese Inschrift kann mit der unseren sicherlich nicht identifiziert werden, ${ }^{7}$ das gleiche gilt für ihren Fundort. Die Base wurde in sekundärer Verwendung gefunden und sicherlich aus einer Stadt, höchstwahrscheinlich aus Brigetio, nach Tát verschleppt. In Pannonien wurden sehr wenige Statuenbasen gefunden, die von städtischen Gemeinschaften oder Stadträten errichtet wurden und nicht einen Kaiser oder die Mitglieder der kaiserlichen Familie darstellen (ILSl 25-26, AIJ 479, RIU 1499). ${ }^{8}$ Paulinus, der früher hier Legatus legionis war, konnte als Patronus von Brigetio mit dieser Statue verehrt werden. Der Name der Stadt wurde sicherlich wegen der Kontamination falsch wiedergegeben. Die Ernennung eines Municipiums (oder einer Colonia) wie Brigetio als $r(e s) p(u b l i c a)$ ist im 3. Jahrhundert sehr oft belegt. ${ }^{9}$

Die Zeichnung und die Inschrift scheinen aber verdächtig zu sein, weil der Text mit wenigen Abweichungen (wie der Erwähnung des Titels vir clarissimus, die erste Zeile fehlt, die zweite Zeile ist stark beschädigt) der Inschrift von Paulinus aus Caerwent (RIB 311) genau folgt (Abb. 3):

[Ti(berio) Claudio] / Paulino / leg(ato) leg(ionis) II / Aug(ustae) proconsul(i) / provinc(iae) Nar/\{r\}bonensis / leg(ato) Aug(usti) pr(o) pr(aetore) provin(ciae) / Lugudunen(sis) / ex decreto / ordinis res / publ(ica) civit(atis) / Silurum.

Es stellt sich sofort die Frage: Wäre es möglich, dass zwei Statuenbasen mit demselben Text in zwei verschiedenen Provinzen (in Britannien und in Pannonien) aufgestellt werden konnten? Die Statuenbase von Caerwent wurde im Jahre 1903 gefunden. ${ }^{10}$ Der Fälscher konnte schon der Zeichnung von F. Haverfield ${ }^{11}$ folgen. Dazu kommt noch, dass die Grabinschrift von Sárisáp (RIU 769) im Jahre 1901 ins Nationalmuseum von Budapest gebracht wurde (Inv.-Nr. 76/1901). Früher war das Steindenkmal in die Mauer des genannten Bades eingemauert, aber es ist auch nicht auszuschließen, dass der Zeichner das Steindenkmal im 19. Jahrhundert gesehen hat. In diesem Fall bleibt es fraglich, ob es sich um eine Fälschung aus dem 20. Jahrhundert handelt oder nicht. Für eine Fälschung würde das überflüssige erste $\mathrm{C}$ der letzten Zeile sprechen, das aus dem Wort civitas der Inschrift von Caerwent stammen kann.

Zusammenfassend kann festgestellt werden, dass die Zeichnung zu den relativ wenigen pannonischen Falsi gehört, oder Ti. Claudius Paulinus eine früher unbekannte Verbindung mit Pannonien hatte. Im ersten Fall bleibt es unsicher, wer, wann (nach 1903) und warum diese Zeichnung und das Heft gefälscht, bzw. kontaminiert hat.

${ }^{6}$ Soproni 1968, 109; Horváth-KelEMEN-Torma 1979, 321-324, Nr. 21.

${ }^{7}$ Herculi / M(arcus) C(- - -) Palternu[s] / v(otum) [s(olvit) l(ibens) $m($ erito)].

${ }^{8}$ Eine Liste der pannonischen Statuenbasen: MrÁv 2003,
${ }^{9}$ Mócsy 1962, 367-384.

${ }^{10}$ HaVerfield 1903a, 420, mit Zeichnung; vgl. HaverFIELD 1903b, 203, Nr. 6; EE 9, 1913, Nr. 1012; AÉp 1903, 232.

${ }^{11}$ HAVERFIELD 1903a. 351-355. 


\section{LITERATUR}

BIRLEY 1982

BIRLEY 2005

HALFMANN 1982

HAVERFIELD 1903a

HAVERFIELD 1903b

HORVÁTH-KELEMEN-TORMA 1979

LEUNISSEN 1989

Mócsy 1959

Mócsy 1962

MRÁv 2003

NÉMETHY 1906

PFLAUM 1978

SOPRONI 1968
= A. R. BIRLEY: Senators from Britain? In: Atti del Colloquio Internazionale AIEGL su Epigrafia e ordine senatorio, Roma, 14-20 maggio 1981, II. Tituli 5. Roma 1982, 531-538.

= A. R. BIRLEY: The Roman government of Britain. Oxford 2005.

$=$ R. Halfmann: Die Senatoren aus den kleinasiatischen Provinzen. In: Atti del Colloquio Internazionale AIEGL su Epigrafia e ordine senatorio, Roma, 14-20 maggio 1981, II. Tituli 5. Roma 1982, 603-650.

= F. HAVERFIELD: A Roman inscription at Caerwent. The Athenaeum Journal, 26. Sept. 1903, 420

= F. HAVERFIELD: Römische Inschriften aus Britannien. Neuer Statthalter. Westd. Korr. 22, 1903/11$12,202-203$.

= I. Horváth-M. Kelemen-I. Torma: Magyarország régészeti topográfiája 5. Komárom megye. Esztergom és dorogi járás. Budapest 1979.

= P. M. M. LEUnISSEN: Konsuln und Konsulare in der Zeit von Commodus bis Severus Alexander (180-235 n. Chr.). Prosopographische Untersuchungen zur senatorischen Elite im römischen Kaiserreich. Dutch Monographs on Ancient History and Archaeology VI. Amsterdam 1989.

= A. Mócsy: Die Bevölkerung Pannoniens bis zu den Markomannenkriegen. Budapest 1959.

= A. MócsY: Ubique res publica: Zu den Autonomiebestrebungen und Uniformierungstendenzen am Vorabend des Dominats. ActaAntHung 10 (1962) 367-384.

= Zs. MrÁv: Die Statuenbase des Philippus Arabs aus Környe. In: Pannonica provincialia et archaeologia. Studia sollemnia auctorum Hungarorum Eugenio Fitz octogenario dedicata. Budapest 2003, 331-368.

= L. NÉMETHY: Esztergom vármegye őstörténete. In: S. Borovszky: Magyarország vármegyéi és városai. Esztergom vármegye. Budapest 1906, 177-191.

= H.-G. Pflaum: Les fastes de la province de Narbonnaise. Paris 1978.

= S. Soproni: TIR L-34. Aquincum-Sarmizegetusa. Budapest 1968. 